\title{
Takatsuki Syndrome - Not Really a Polyneuropathy, Organomegaly, Endocrinopathy, M-spike, and Skin Change!!!
}

\begin{abstract}
Polyneuropathy, organomegaly, endocrinopathy, M-spike, and skin changes (POEMS) is a rare multisystem disorder, with its prevalence in India being 1 in 330,000. It is a demyelinating neuropathy accompanied by dermatoendocrine syndrome. The cause of POEMS is unknown. The diagnosis of POEMS syndrome requires two mandatory criteria plus more than one major and more than one minor criterion. A middle-aged male with no known comorbidities from South India presented with complaints of burning micturition, increased frequency of micturition, and pain in the abdomen for 3 weeks. The patient was evaluated earlier at another center with a rise in serum creatinine from 1.9 to $2.9 \mathrm{mg} / \mathrm{dl}$ within a span of 3 days. The patient had pleural effusion, hypercalcemia, renal failure, and splenomegaly with lytic lesions. Due to the presence of typical skin lesions with polyneuropathy, workup for POEMS was carried out. Positron-emission tomography scan and vascular endothelial growth factor levels were checked and fulfilled the criteria. Early treatment was initiated, and POEMS has a good prognosis. As POEMS is very rare, early diagnosis improves the outcome.
\end{abstract}

Keywords: Multiple myeloma, POEMS, skin changes, vascular endothelial growth factor

\section{Introduction}

Polyneuropathy,

organomegaly,

endocrinopathy, M-spike, and skin changes (POEMS) or Crow-Fukase syndrome or osteosclerotic myeloma is a rare multisystem disorder, with a prevalence in India being 1 in 330,000 . It is most prevalent in Japan. The peak incidence is in the fifth and sixth decades of life, unlike multiple myeloma which has a peak incidence in the seventh and eighth decades of life. Initially, it was known as osteosclerotic myeloma and the term POEMS was coined by Bardwick in 1980. It is often confused with chronic inflammatory demyelinating polyneuropathy (CIDP). ${ }^{[1]}$ It is a demyelinating neuropathy accompanied by dermatoendocrine syndrome. ${ }^{[2]}$ The cause of POEMS is unknown. However, it is observed that plasma and serum vascular endothelial growth factor (VEGF) levels are markedly elevated in those cases. It is postulated that VEGF is secreted from plasma cells and platelets promoting vascular permeability, angiogenesis, and macrophage migration, potentially resulting in arterial obliteration. VEGF could be

This is an open access journal, and articles are distributed under the terms of the Creative Commons Attribution-NonCommercial-ShareAlike 4.0 License, which allows others to remix, tweak, and build upon the work non-commercially, as long as appropriate credit is given and the new creations are licensed under the identical terms.

For reprints contact: WKHLRPMedknow_reprints@wolterskluwer.com the cause for organomegaly, edema, and skin lesions. It is stated to play a role in microthrombosis occurring in POEMS syndrome. Diagnostic criteria for POEMS syndrome include the following:

- Mandatory criteria: (1) polyneuropathy and (2) monoclonal plasma cell proliferative disorder

- Major criteria: (1) Castleman disease, (2) sclerotic bone lesions, and (3) elevated vascular endothelial growth factor levels

- Minor criteria: (1) organomegaly, (2) extravascular volume overload, (3) endocrinopathy, (4) skin changes such as hyperpigmentation, hypertrichosis, and vascular skin changes, (5) papilledema, and (6) thrombocytosis.

The diagnosis of POEMS syndrome requires two mandatory criteria plus more than one major and more than one minor criteria. Castleman disease is a variant of POEMS syndrome that occurs without evidence of a clonal plasma cell disorder. As it has varied presentations, early diagnosis can be difficult and can be missed.

\section{Case Report}

A 54-year-old male with no known comorbidities from Kannur in South India

How to cite this article: Vineeth VK, Shetty V. Takatsuki syndrome-Not really a polyneuropathy, organomegaly, endocrinopathy, M-spike, and skin change!!! Indian J Med Paediatr Oncol 2020;41:608-11.

\section{Vashe Keshava Vineeth ${ }^{1}$, Vijith Shetty ${ }^{2}$}

${ }^{I}$ Department of General Medicine, Unit 1V, Christian Medical College, Vellore, Tamil Nadu, India, ${ }^{2}$ Department of Medical Oncology, K. S. Hegde Medical Academy, NITTE Deemed to be University, Deralakatte, Karnataka, India

Submitted: 09-Jun-2019 Revised: 02-Jan-2020

Accepted: 19-Jan-2020 Published: 29-Aug-2020

Address for correspondence: Dr. Vijith Shetty, Department of Oncology, OPD No 50, K. S. Hegde Hospital, Deralakatte - 575018 Karnataka, India.

E-mail:drvijithshetty@gmail. com

Access this article online Website: www.ijmpo.org

DOI: 10.4103/ijmpo.ijmpo_137_19 Quick Response Code:

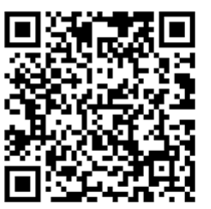


presented with complaints of burning micturition, increased frequency, and pain in the abdomen for 3 weeks. He had complaints of easy fatigability and unable to walk properly for 2 months. The patient was evaluated at an outside center with the rise in serum creatinine from 1.9 to $2.9 \mathrm{mg} / \mathrm{dl}$ in 3 days. He presented to our hospital for further workup. Initially, we had a suspicion of acute kidney injury (AKI) with cause to be worked up.

On examination, he had tachycardia with vitals stable. Systemic examination revealed the spleen to be palpable with basal consolidation. It was noted that his ankle jerk was sluggish.

Pallor was present, but no icterus, cyanosis, and pedal edema were noted.

Abominal examination revealed: Diffuse tenderness, and the spleen was just palpable.

Cardiovascular system was normal. Respiratory system - reduced breath sounds were noted in the bilateral base. Right-sided basal crepitations were heard.

Peripheral nervous system, bilateral ankle jerk was sluggish. Rest was normal.

On local examination, multiple erythematous plaques over the scalp and base of the chest and the abdomen with white nails and plethora were observed.

The patient's relatives reported a history of receiving local corticosteroids for a short duration of time for the skin lesions.

Initial blood reports showed mild anemia, high erythrocyte sedimentation rate, AKI with life-threatening hypercalcemia, high serum calcium of $17.6 \mathrm{mg} / \mathrm{dl}$, and liver function test showing altered AG ratio. Hence, we worked up for the causes of the same. Thyroid-stimulating hormone and parathyroid hormone were low with values of 0.233 and $6.90 \mathrm{pg} / \mathrm{ml}$, respectively. Urine microscopy showed features suggestive of urinary tract infection (UTI), and urine culture was sent which subsequently showed no growth. He also had an lower respiratory tract infection with a chest X-ray showing right-sided nonhomogeneous opacity with pleural effusion. Initially, the patient was suspected with AKI, UTI, hypercalcemia under evaluation, and right lower lobe consolidation. Initially, the patient was managed conservatively with intravenous (IV) fluids and IV antibiotics which were later shifted to higher spectrum antibiotics such as meropenem, after which, fever spikes reduced. In view of the hypercalcemia, the patient was further worked up with suspicion toward multiple myeloma. Serum electrophoresis was done, which showed M-band. X-ray skull lateral view showed multiple lytic lesions [Figure 1].

Serum-free kappa light chain was normal, but serum-free lambda was $25.1 \mathrm{mg} / \mathrm{dl}$ (elevated) with altered free kappa lambda ratio. Beta-2 macroglobulin was 5.57 which was elevated indicating stage 3 of the disease. In view of right-sided basal crepitations, computed tomography (CT) of the thorax was done, which showed bilateral lower lobe lung parenchymal opacities with pleural effusion and multiple lytic lesions in the spine. As the spleen was just palpable with diffuse tenderness, $\mathrm{CT}$ of the abdomen was done which showed thickening with air density pockets in the right psoas and iliacus muscle and splenomegaly and multiple lytic lesions in the vertebral column with pathological fractures [Figures 2-5]. As this patient also had significant skin manifestations, suspicion toward osteosclerotic myeloma/Crow-Fukase syndrome was made. Hence, the patient was worked up [Figure 6].

Bone marrow was done which showed plasma cell dyscrasia with hypercellular marrow with sheets of plasma cells $(50 \%-60 \%)$ including immature forms. The FISH study was done on the marrow which showed no chromosomal abnormalities. Vascular endothelial growth factor was checked which was found to be elevated out of proportion with the value being $547 \mathrm{pg} / \mathrm{ml}$. Further workup by means of nerve conduction study showed asymmetrical right more than left axonal sensorimotor peripheral neuropathy in both upper and lower limbs.

The patient was managed with adequate hydration, IV meropenem, corticosteroids, and cyclophosphamide. Once fever spikes and infection reduced and after bone marrow aspiration was taken, the patient received 2 doses of bortezomib $2 \mathrm{mg} \mathrm{S} / \mathrm{C}$ with a gap of 7 days and zoledronic acid after the second dose.

The recovery was phenomenal with creatinine lowering to $1.37 \mathrm{mg} / \mathrm{dl}$ and calcium to $9.6 \mathrm{mg} / \mathrm{dl}$. The patient's appetite improved and the patient also received palliative radiotherapy of $30 \mathrm{Gys} / 10$ fractions in a span 2 weeks during the stay. In view of radio imaging showing multiple lytic lesions and multiple air pockets, suspicion of plasmacytoma was made, and positron-emission

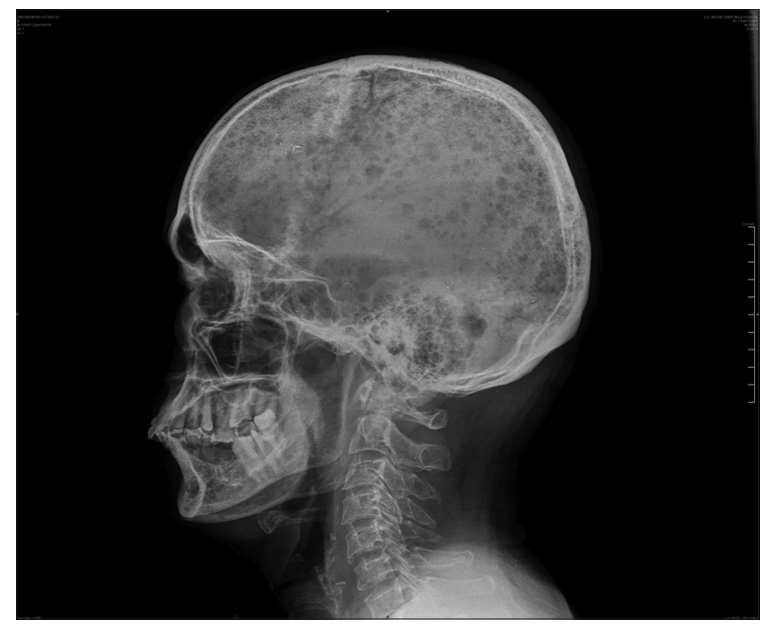

Figure 1: X-ray skull showing lytic lesions 


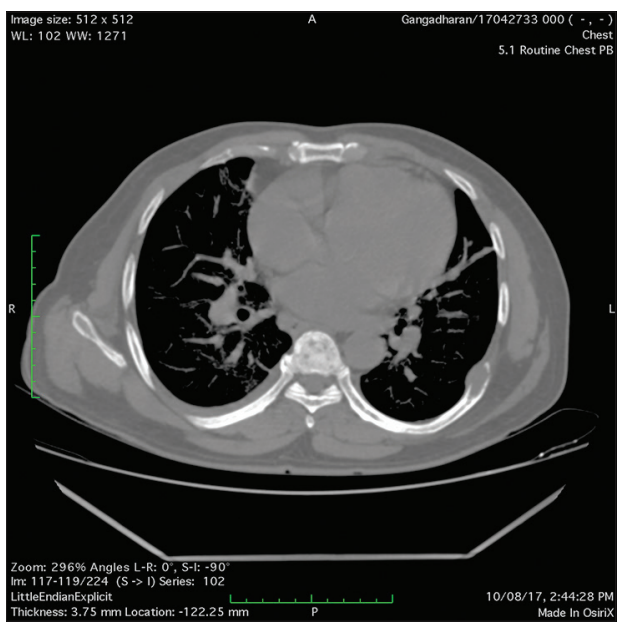

Figure 2: Computed tomography thorax was done which showed bilateral lower lobe lung parenchymal opacities with pleural effusion and multiple lytic lesions in the spine

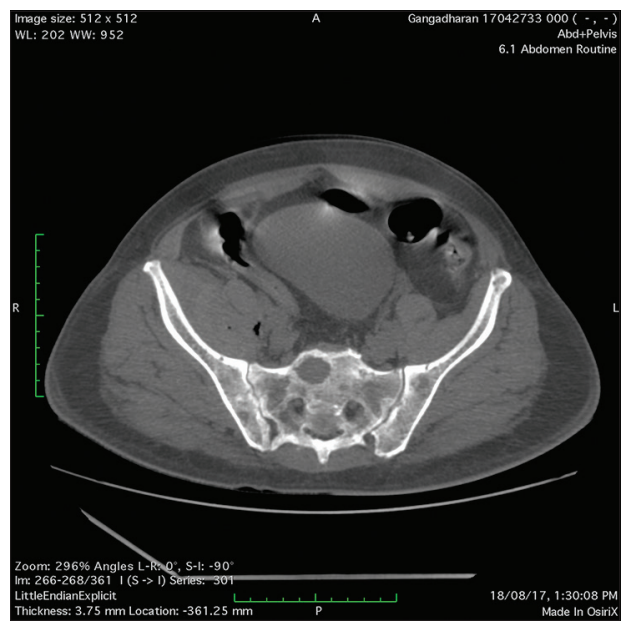

Figure 4: Computed tomography of the abdomen showing splenomegaly

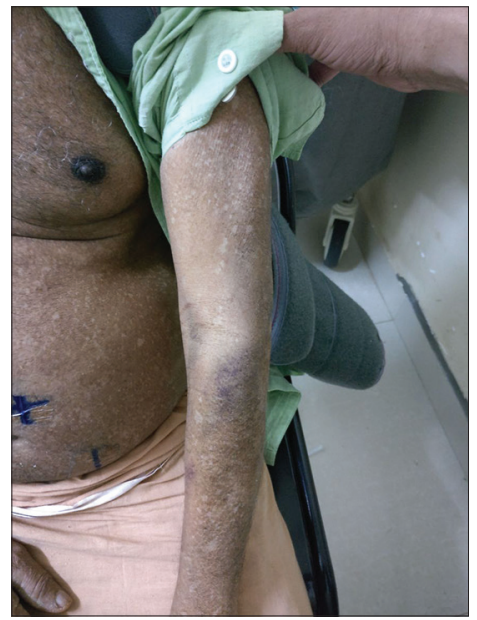

Figure 6: Skin changes typical of polyneuropathy, organomegaly, endocrinopathy, M-spike, and skin changes

tomography scan showed low-grade metabolic activity in multiple sites with multiple extensive lytic lesions confirming POEMS [Figure 7]. The patient was diagnosed

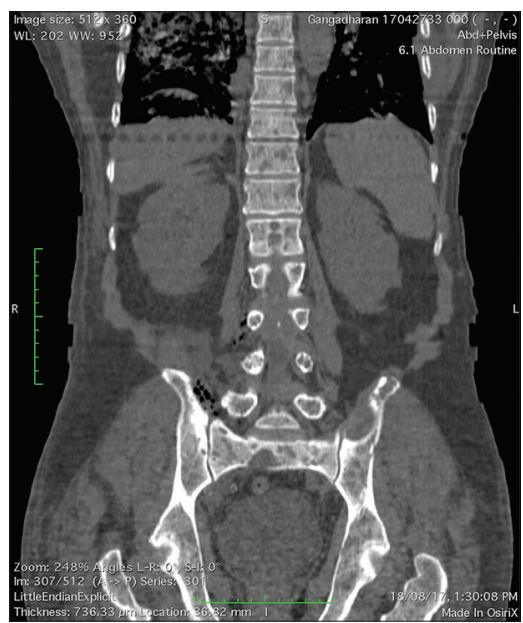

Figure 3: Computed tomography abdomen showing organomegaly with multiple lytic lesions over the spine

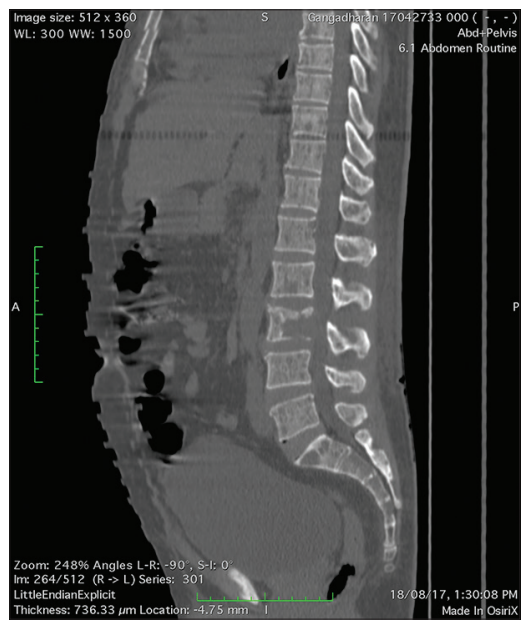

Figure 5: Computed tomography of the abdomen showed thickening with air density pockets in the right psoas and iliacus muscle and splenomegaly and multiple lytic lesions in the vertebral column with pathological fractures

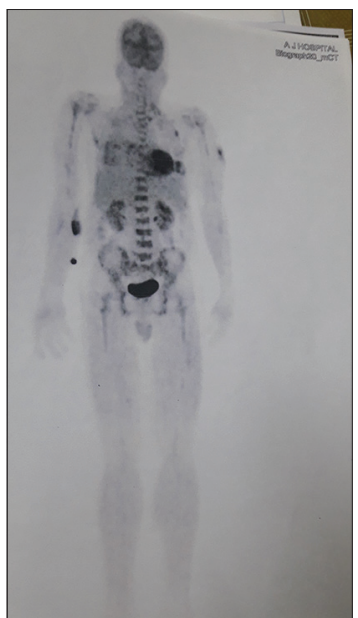

Figure 7: Positron-emission tomography scan showed low-grade metabolic activity in multiple sites with multiple extensive lytic lesions confirming polyneuropathy, organomegaly, endocrinopathy, M-spike, and skin changes

to have POEMS fulfilling both mandatory criteria polyneuropathy and monoclonal plasma cell proliferative 
disorder. Two major criteria fulfilled were sclerotic bone lesion and elevated VEGF And four minor criteria fulfilled were splenomegaly, pleural effusion, endocrinopathy, and skin changes, although for the diagnosis of POEMS required only two mandatory criteria and more than one major criterion. The patient was discharged after 1 month of hospital stay with dramatic improvement with antibiotics, antifungal, antiviral, corticosteroids, and cyclophosphamide. The patient came for follow-up after 20 days and received the second cycle chemotherapy, with serum creatinine touching below baseline and skin changes almost disappearing. The patient has been advised for a timely follow-up in view of the good prognosis with the disease.

\section{Discussion}

The mode of treatment for POEMS is based on whether the patient has limited or widespread sclerotic bone lesions. Chemotherapy with autologous hematopoietic cell transplantation should be considered for young patients. It is very important to distinguish POEMS from CIDP as both will have peripheral neuropathy. POEMS show higher degrees of axonal degeneration and more epineurial neovascularization than CIDP. Our patient was treated with bortezomib and radiation which is most accepted than treatment with melphalan. We had even advised hematopoietic stem cell transplantation as the median survival of POEMS is 13.7.

In a study by Daniele $\mathrm{C}$ and Eva $\mathrm{LF}^{[3]}$ discussed the importance of radiotherapy in patients of POEMS with no bone marrow involvement and not more than three bone lesions. However, on diffuse involvement, the benefit is not explained.

A study by Dispenzieri $\mathrm{A}^{[4]}$ discussed that the need for prompt treatment directed at the underlying plasma cell clone produces a dramatic response in majority of patients. They suggested that for patients with disseminated disease, high-dose chemotherapy with peripheral blood transplantation has yielded durable benefits and radiotherapy is effective for a localized disease.

\section{Conclusion}

Patients with POEMS syndrome present with a complex conglomerate of symptoms, signs, and objective abnormalities, making the diagnosis, management, and follow-up a challenge. We highlight this rare syndrome which is very uncommon in India, which requires prompt diagnostic workup with a multidisciplinary team of doctors. Early diagnosis and treatment can improve the quality of life as the overall survival rate is good with POEMS.

\section{Declaration of patient consent}

The authors certify that they have obtained all appropriate patient consent forms. In the form the patient(s) has/have given his/her/their consent for his/her/their images and other clinical information to be reported in the journal. The patients understand that their names and initials will not be published and due efforts will be made to conceal their identity, but anonymity cannot be guaranteed.

\section{Acknowledgment}

The author thank Allmighty, institution, and the patient for the support.

\section{Financial support and sponsorship}

Nil.

\section{Conflicts of interest}

There are no conflicts of interest.

\section{References}

1. Nyunt WW, Remli R, Abdul Muttlib FA, Leong CF, Masir N, Tumian NR, et al. POEMS syndrome. Malays J Pathol 2017;39:297-303.

2. Angela D, Morie GA. POEMS syndrome. Orphanet 2005;1:1-14.

3. Daniele C, Eva LF. POEMS syndrome treated with radiotherapy and literature. Cancer Ther Oncol 2017;4:1-5.

4. Dispenzieri A. How I treat POEMS syndrome. Blood 2012;119:5650-8. 